\title{
Virtual Hawking Radiation
}

\author{
Walter Goldberger ${ }^{1}$ and Ira Z. Rothstein ${ }^{2}$ \\ ${ }^{1}$ Department of Physics, Yale University, New Haven, Connecticut 06511, USA \\ ${ }^{2}$ Department of Physics, Carnegie Mellon University, Pittsburgh, Pennsylvania 15213, USA
}

(Received 13 July 2020; accepted 13 October 2020; published 19 November 2020)

\begin{abstract}
We consider the effects of off shell Hawking radiation on scattering processes involving black holes coupled to quantum fields. The focus here is on the case of gravitational scattering of a scalar field mediated by the exchange of virtual Hawking gravitons from a four-dimensional Schwarzschild black hole. Our result is obtained in the context of a worldline effective field theory for the black hole and is valid in the semiclassical limit where the Schwarzschild radius $r_{s}$ is larger than the Planck length $1 / m_{\mathrm{Pl}}$. In addition, we assume that four-momentum exchange $q$ is smaller than $r_{s}^{-1}$ and that the incoming particle has energy larger then the black hole's Hawking temperature. The inelastic cross section we obtain is a new, leadingorder quantum gravity effect, arising at the same order in $q^{2} / m_{\mathrm{Pl}}^{2}$ as the well-understood one-loop graviton vacuum polarization corrections to gravitational scattering between massive particles.
\end{abstract}

DOI: 10.1103/PhysRevLett.125.211301

Introduction.-While quantum gravity at the Planck scale still remains a mystery, it is commonly believed that the low-energy gravitational $S$ matrix is by now completely understood. In particular, the UV divergences that arise in the calculation of scattering amplitudes containing either on shell or virtual gravitons can be treated systematically by the standard tools of effective field theory (EFT) [1]. For instance, the graviton-graviton elastic scattering amplitude in pure gravity is finite at one loop [2], while at two loops [3] it exhibits logarithmic divergences that can be absorbed into the Einstein-Hilbert action supplemented by higherderivative operators, schematically

$$
S=-2 m_{\mathrm{Pl}}^{2} \int d^{4} x \sqrt{g}\left[R+\frac{1}{m_{\mathrm{Pl}}^{4}} R_{\mu \nu \alpha \beta}^{3}+\cdots\right],
$$

with higher-order terms suppressed by more powers of $E^{2} / m_{\mathrm{Pl}}^{2} \ll 1$ at low energies. [We define $m_{\mathrm{Pl}}^{2}=1 / 32 \pi G_{N}$. In Eq. (1), curvature squared terms can be either removed by field redefinitions of the graviton or traded for topological terms that have no effect on perturbative observables.] Although this methodology yields well-defined predictions in the low-energy approximation of quantum gravity for processes involving elementary particles coupled to gravitons, the computation of the quantum gravity $S$ matrix with black hole asymptotic states has yet to be accomplished, even at energies below the Planck

Published by the American Physical Society under the terms of the Creative Commons Attribution 4.0 International license. Further distribution of this work must maintain attribution to the author(s) and the published article's title, journal citation, and DOI. Funded by SCOAP ${ }^{3}$. scale. (The meaning of "asymptotic state" for the case of black holes that decay via the emission of Hawking radiation will be further discussed in the next section.) In this case, there are new nonperturbative quantum effects associated with Hawking radiation [4], which play a crucial role. The emission of on shell Hawking radiation from fixed black hole backgrounds has been thoroughly studied [5]. However, the effects of virtual Hawking modes represent another source of quantum gravity corrections whose analysis is still unchartered territory that should be amenable to a field theoretic treatment.

In a recent paper [6], we have introduced an effective field theory framework designed to calculate quantum corrections to processes involving black holes interacting through the exchange of long wavelength fields. It builds on methods described in $[7,8]$ to treat the effects of classical absorption by the horizon of a black hole within a worldline effective theory [9] of the gravitational dynamics of compact objects. The main idea of [6-8] is that emission and absorption of quanta by the horizon are due to horizon localized degrees of freedom that couple to external fields. In the limit where the black hole radius $r_{s}=2 G_{N} M$ is small, these localized modes are described by a quantum mechanical $(0+1)$ theory whose correlation functions can be extracted model independently, by matching on shell emission and absorption processes in the full semiclassical black hole spacetime. The same correlators can then be used to predict observables where the black hole horizon exchanges off shell modes with other objects, for instance, the classical dissipation of energy in the binary dynamics of comparable mass black holes $[7,8]$.

A somewhat counterintuitive property of the black hole worldline correlators obtained in [6] is that, for black holes in the Unruh state [10] (i.e., black holes formed from the 
gravitational collapse of matter), Hawking emission is not suppressed by powers of $\hbar$. Instead the Hawking response is enhanced at low frequency relative to classical absorption by the horizon. This is tied to the well-known fact that the distribution of emitted Hawking quanta from a semiclassical black hole is independent of $\hbar$, as well as to detailed balance arguments for black holes in thermal equilibrium [11] with a radiation bath at the Hawking temperature $T_{H}=\hbar / 4 \pi r_{s}$. While the Wightman functions calculated in [6] are themselves not suppressed by powers of $\hbar$ or, equivalently, $1 / m_{\mathrm{Pl}}$, the causal (retarded) Green's functions in the Unruh state were shown to be insensitive to Hawking radiation, at least up to corrections from bulk interactions of the fields propagating around the black hole. As a consequence, there are no observable corrections to classical processes (e.g., binary dynamics) from Hawking modes, as classical intuition would suggest.

On the other hand, there are processes, such as quantum mechanical scattering of matter fields incident on the black hole, that depend on worldline correlators other than the causal two-point function. In this case, the effects of (off shell) Hawking radiation do not cancel. It is then interesting to ask how their magnitude compares to the more familiar loop corrections based on a perturbative treatment of Eq. (1). To address this, in this Letter, we consider an inelastic scattering process where a quantum field (for simplicity, a complex scalar $\phi$ ) minimally coupled to gravity scatters off a black hole via the exchange of an off shell Hawking graviton mode. We obtain a well-defined (calculable) prediction for the inelastic scattering cross section, which is of the same order in $1 / m_{\mathrm{Pl}}^{2}$ as the canonical one-loop quantum gravity corrections to the elastic scattering cross section corrections arising from interference with single graviton exchange.

In next section, we summarize the EFT setup, including the relevant hierarchy of scales in which our description holds, as well as the systematics of the power counting. Details of the matching calculation needed to extract the relevant worldline correlators can be found in [12]. We then compute the leading-order inelastic process induced by Hawking graviton exchange and compare to the elastic scattering process. Our main result is given in Eq. (18). In the Conclusions, we summarize and outline directions for future work.

The EFT formalism and power counting.-We are interested in scattering processes where matter fields scatter gravitationally off a quantum mechanical black hole. To be definite, we consider the case of a complex scalar field $\phi$ coupled minimally to gravity,

$$
S=\int d^{4} x \sqrt{g}\left(g^{\mu \nu} \partial_{\mu} \phi^{\dagger} \partial_{\nu} \phi-m^{2} \phi^{\dagger} \phi\right) .
$$

This action, along with the Einstein-Hilbert term $S_{\mathrm{EH}}=-2 m_{\mathrm{Pl}}^{2} \int d^{4} x \sqrt{g} R+\cdots$, is sufficient to study the effects of quantum gravity as long as we are interested in processes where all energy and momentum scales, and there fore the curvature, are small compared to the Planck scale.

Of particular interest here is the case where the scalar $\phi$ and the graviton field $h_{\mu \nu}$ propagate in the background of a black hole solution to Einstein's equations. We take the case of Schwarzschild black holes for simplicity and assume that the curvature at the horizon is small in Planck units. Then the interactions of scalar and graviton can be analyzed in a derivative expansion of the action about the Schwarzschild background that is both systematic and tractable.

In order to sidestep the technical difficulties of quantizing the graviton in the full Schwarzschild background, including the effects of Hawking radiation from the black hole horizon, we will use the effective field theory methods developed in [6-9]. In this EFT, one begins by first considering the black hole in the point particle approximation. In so doing, we have integrated out all of the internal dynamics, with finite size effects systematically accounted for by including all higher-dimensional operators (composed of the curvature, as well as other fields) that are consistent with symmetries of the underlying UV theory. This will not suffice, however, to describe either Hawking radiation or absorption, which imply the existence of gapless degrees of freedom associated with the dynamics of the horizon.

To account for these gapless modes in a modelindependent way, we introduce a quantum mechanical Hilbert space of states localized on the black hole worldline coordinate $x^{\mu}(\tau)$. In this description, the semiclassical black hole with mass $M \gg m_{\mathrm{Pl}}$ corresponds to a highly excited state $|M\rangle$ where the mass is hierarchically larger than $1 / r_{s}$. In the absence of couplings to, e.g., external gravitational or electromagnetic interactions, the state $|M\rangle$ is an eigenstate of the black hole Hamiltonian $H_{o}$. The external fields couple to composite worldline operators made out of the black hole internal degrees of freedom. Absent a specific model, we classify these operators by their quantum numbers under $\mathrm{SO}(3)$ isometries of the Schwarzschild geometry and couple them to external fields in all ways consistent with symmetry. For instance, at leading order in the multipole expansion, the tidal gravitational response is accounted for by including $\ell=2$ (quadrupole) operators $Q_{a b}^{E}(\tau), Q_{a b}^{B}(\tau)$ of electric and magnetic parity, whose gravitational interactions are encoded in the action

$S_{\mathrm{int}}=-\int d \tau Q_{a b}^{E}(\tau) E^{a b}[x(\tau)]-\int d \tau(\tau) Q_{a b}^{B}(\tau) B^{a b}[x(\tau)]$.

Here, the indices $a, b=1,2,3$ refer to a spatial frame $e_{\mu}^{a}(\tau)$ that describes the orientation of the black hole relative to the ambient space. By definition this frame obeys the constraints $v^{\mu} e_{\mu}^{a}=0$, and 


$$
\begin{aligned}
& g^{\mu \nu} e_{\mu}^{a} e_{\nu}^{a}=-\delta^{a b}, \\
& \delta_{a b} e_{\mu}^{a} e_{\nu}^{b}=g_{\mu \nu}-v_{\mu} v_{\nu},
\end{aligned}
$$

with $v^{\mu}=d x^{\mu} / d \tau$ the four velocity of the black hole. The projected curvature tensors are $E^{a b}=e_{\mu}^{a} e_{\nu}^{b} E^{\mu \nu}$ and $B^{a b}=e_{\mu}^{a} e_{\nu}^{b} B^{\mu \nu}$, where the electric and magnetic components of the curvature tensor are

$$
\begin{aligned}
& E_{\mu \nu}=R_{\mu \alpha \nu \beta} v^{\alpha} v^{\beta}, \\
& B_{\mu \nu}=\tilde{R}_{\mu \alpha \nu \beta} v^{\alpha} v^{\beta}=\frac{1}{2} \epsilon_{\mu \alpha \rho \sigma} R_{\beta \nu}^{\rho \sigma} v^{\alpha} v^{\beta} .
\end{aligned}
$$

(In practice, the Ricci curvature parts of $R_{\mu \nu \alpha \beta}$ can be removed by field redefinitions of the graviton, so do not have any physical effects.)

The validity of the effective worldline description is limited to the regime where the black hole interacts with probes whose typical frequency (or wave number) $\omega$ lies in the range

$$
\tau_{\mathrm{BH}}^{-1} \ll \omega \ll 1 / r_{s},
$$

where the upper bound arises as a consequence of the point particle approximation $\left(r_{s}=2 G_{N} M\right.$ is the Schwarzschild radius), and the lower bound ensures that we are looking at timescales short compared to the Page time $\tau_{\mathrm{BH}} \sim M^{3} / m_{\mathrm{Pl}}^{2}$, so that we can ignore the backreaction due to the evaporation process. We also take the black hole to be semiclassical, with mass $M \gg m_{\mathrm{Pl}}$.

As explained in [6,7], physical processes involving the black hole coupled to other fields are described in this EFT in terms of the Wightman functions of worldline operators such as $Q_{a b}^{E}(\tau)$ and $Q_{a b}^{B}(\tau)$, which can be obtained by a matching calculation to the full theory of fields propagating in the black hole spacetime. For a nonrotating black hole, the two-point Wightman functions in the frame where the black hole is at rest then take the form

$$
\left\langle M\left|Q_{a b}^{E, B}(t) Q_{c d}^{E, B}(0)\right| M\right\rangle=\langle a, b \mid c, d\rangle \int_{-\infty}^{\infty} \frac{d \omega}{2 \pi} e^{-i \omega t} A_{+}^{E, B}(\omega),
$$

where $\langle a, b \mid c, d\rangle=\frac{1}{2}\left[\delta_{a c} \delta_{b d}+\delta_{a d} \delta_{b c}-\frac{2}{3} \delta_{a b} \delta_{c d}\right]$ is the identity operator on the space of $\ell=2$ tensors.

The Wightman functions $A_{+}^{E, B}(\omega)$ are obtained by a matching calculation described in [7], which compares the EFT to multiparticle scattering and production probabilities $[13,14] p(n \rightarrow m)$ for the black hole in the Unruh state [10] (corresponding to a noneternal black hole, formed by realistic gravitational collapse). Adapting the methods of [7] to the case of gravitons, we find in the Appendix of [12] that, to leading order in $r_{s} \omega \ll 1$,

$$
A_{+}^{E}(\omega)=A_{+}^{B}(\omega) \approx \frac{r_{s}^{5}}{360 \pi G_{N}} .
$$

In particular, the presence of nonvanishing response at $\omega<$ 0 accounts for emission of Hawking gravitons near the horizon, while the $\omega>0$ branch represents absorption.

In this Letter, we will use the EFT to analyze the inelastic scattering of matter fields, represented here by the complex scalar $\phi$ of Eq. (2) incident on a black hole with mass $M \gg m$. Since the case where the scalar field has negligible mass compared to the Hawking temperature, $T_{H}=\left(4 \pi r_{s}\right)^{-1}$, corresponding to absorption and reemission of on shell scalars, is well understood [13], we focus instead on the limit $m \gg T_{H}$. In this regime, the dominant inelastic process is through the exchange of off shell Hawking gravitons between the scalar and the black hole. Alternatively, one could also study the limit where the incoming scalar has energy $E_{\phi} \gg T_{H}$, where again the scattering process is dominated by graviton exchange. However, in order to remain within the regime of validity of the EFT, we take the typical momentum transfer $q$ (or, equivalently, the impact parameter $b \sim 1 / q$ ) to lie in the region defined by Eq. (6). Thus, to ensure the validity of the EFT, we assume the following hierarchy of kinematic scales:

$$
M \gg E_{\phi} \gg T_{H} \gg q \sim b^{-1} \gg \tau_{\mathrm{BH}}^{-1} .
$$

Scattering by off shell Hawking radiation.-We now compute the inelastic process $\phi(p)+\mathrm{BH}_{M} \rightarrow \phi\left(p^{\prime}\right)+X$, where a scalar field scatters off a heavy Schwarzschild black hole. Because of the presence of the horizon, the scalar can tidally exchange energy and momentum with the black hole. In the Unruh state, the exchanged energy can be of either sign, due to the possibility that $\phi$ absorbs a virtual Hawking mode emitted by the black hole.

In the EFT, the inclusive probability is given in Fig. 1, where we sum over the unobserved internal states $X$ of the final black hole. The interaction between the black hole and $\phi$ is mediated by graviton exchange. Linearizing about flat space, $g_{\mu \nu}=\eta_{\mu \nu}+h_{\mu \nu} / m_{\mathrm{Pl}}$, the relevant term in Eq. (2) is

$$
\mathcal{L}_{\text {int }}=-\frac{h^{\mu \nu}}{m_{\mathrm{Pl}}}\left[\partial_{\mu} \phi^{\dagger} \partial_{\nu} \phi-\frac{1}{2} \eta_{\mu \nu}\left(|\partial \phi|^{2}-m^{2} \phi^{\dagger} \phi\right)\right] .
$$

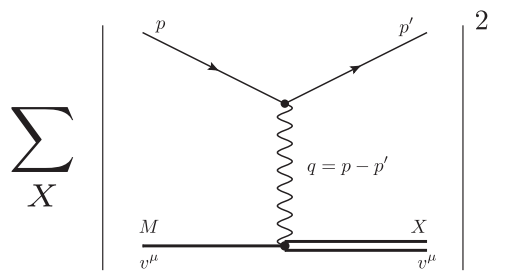

FIG. 1. Leading-order gravitational inelastic scattering of a scalar field incident on a semiclassical Schwarzschild black hole. 
Our calculation is performed in Feynman gauge, where the propagator of the exchanged graviton is $\frac{i}{q^{2}} P_{\mu \nu ; \alpha \beta}$, with

$$
P_{\mu \nu ; \alpha \beta}=\frac{1}{2}\left[\eta_{\mu \alpha} \eta_{\nu \beta}+\eta_{\mu \beta} \eta_{\nu \alpha}-\eta_{\mu \nu} \eta_{\alpha \beta}\right] .
$$

In the rest frame of the black hole $v^{\mu}=(1,0)$, the amplitude to leading order in the EFT of Eq. (3) takes the form $\left(q=p-p^{\prime}\right.$ is the momentum transfer)

$i \mathcal{A}_{X}=-\frac{1}{2 m_{\mathrm{Pl}}^{2}} \frac{i}{q^{2}} \int d \tau e^{-i q v \tau}\left\langle X\left|Q_{a b}^{E}(\tau)\right| M\right\rangle \mathcal{A}_{a b}^{E}+$ mag.,

where the tensors $\mathcal{A}_{a b}^{E}=e^{\mu}{ }_{a} e^{\nu}{ }_{b} \mathcal{A}_{\mu \nu}^{E}$ are given by

$$
\begin{aligned}
\mathcal{A}_{\mu \nu}^{E}= & {[(v p) q-(v q) p]^{\mu}[(v p) q-(v q) p]^{\nu} } \\
& -\frac{1}{2} m^{2}\left[q_{\mu}^{\perp} q_{\nu}^{\perp}+(v q)^{2} \eta_{\mu \nu}^{\perp}\right], \\
\mathcal{A}_{\mu \nu}^{B}= & \epsilon_{\mu \alpha \rho \sigma} v^{\alpha} p^{\rho} q^{\sigma}[(v q) p-(v p) q]^{\nu} \\
& +\frac{1}{2} m^{2}(v q) \epsilon^{\mu \nu \rho \sigma} v_{\rho} q_{\sigma} .
\end{aligned}
$$

$\left[q_{\perp}^{\mu}=q^{\mu}-(v q) v^{\mu}, \eta_{\perp}^{\mu \nu}=\eta^{\mu \nu}-v^{\mu} v^{\nu}\right.$.] In order to perform the tensor contractions, we have used the package [15].

Summing over the final states $X$ and assuming unitarity of the black hole quantum mechanics, $\sum_{X}|X\rangle\langle X|=1$, the inclusive squared amplitude breaks up into electric and magnetic contributions

$$
\sum_{X}\left|\mathcal{A}_{X}\right|^{2}=\left|\mathcal{A}_{E}\right|^{2}+\left|\mathcal{A}_{B}\right|^{2},
$$

which depend on the two-point Wightman functions defined in Eq. (7) (note that, by parity invariance, the mixed correlator $\left\langle Q^{E} Q^{B}\right\rangle$ vanishes). For example, the electric term in the case of zero black hole spin is

$$
\left|\mathcal{A}_{E}\right|^{2}=\frac{1}{4 m_{\mathrm{Pl}}^{4}} \frac{T}{q^{4}} A_{+}^{E}(\omega)\langle a, b \mid c, d\rangle \mathcal{A}_{a b}^{E} \mathcal{A}_{c d}^{E},
$$

and similar for the magnetic piece. The timescale $T=2 \pi \delta(\omega \rightarrow 0)$ is an arbitrary IR cutoff associated with time translation invariance, which will not appear in physical observables. We find, from Eqs. (13),

$$
\begin{aligned}
\left|\mathcal{A}_{E}\right|^{2} \approx & \frac{T A_{+}^{E}(q v)}{6 m_{\mathrm{Pl}}^{4}}\left[(v p)^{4}-m^{2}(v p)^{2}\left(1-\frac{3}{2} \frac{(v q)^{2}}{q^{2}}\right)\right. \\
& \left.+\frac{1}{4} m^{4}\left(1+3 \frac{(v q)^{4}}{q^{4}}\right)\right], \\
\left|\mathcal{A}_{B}\right|^{2} \approx & \frac{T A_{+}^{B}(q v)}{8 m_{\mathrm{Pl}}^{4}}\left[(v p)^{4}-m^{2}(v p)^{2}\left(1-2 \frac{(v q)^{2}}{q^{2}}\right)\right. \\
& \left.-m^{4} \frac{(v q)^{2}}{q^{2}}\left(1-\frac{(v q)^{2}}{q^{2}}\right)\right],
\end{aligned}
$$

where we drop terms subleading in powers of the momentum transfer $q$. The resulting black hole frame differential cross section for inelastic scattering is then

$$
\begin{aligned}
& \frac{d^{3} \sigma}{d q^{2} d(q v)} \approx \frac{7 G_{N} r_{s}^{5}}{270 \pi\left[(v p)^{2}-m^{2}\right]}\left[(v p)^{4}-m^{2}(v p)^{2}\right. \\
& \left.\left(1-\frac{12}{7} \frac{(v q)^{2}}{q^{2}}\right)+\frac{1}{7} m^{4}\left(1-3 \frac{(v q)^{2}}{q^{2}}+6 \frac{(v q)^{4}}{q^{4}}\right)\right] .
\end{aligned}
$$

It is useful to compare the magnitude of this result with the leading-order cross section for Newtonian potential scattering off the black hole. In the black hole rest frame, this is given by

$$
\frac{d \sigma_{N}}{d q^{2}}=\frac{4 \pi r_{s}^{2}}{q^{4}} \frac{\left[(v p)^{2}-\frac{1}{2} m^{2}\right]^{2}}{(v p)^{2}-m^{2}} .
$$

To compare this to the off shell Hawking process, we would need to integrate Eq. (18) over the region $-\infty<v q<\infty$. Although the EFT breaks down when the magnitude of $q v$ is of order $r_{s}$, we expect, by unitarity, that the integral over the form factors $A_{+}^{E, B}(q v)$ is finite and dominated by scales near $q v$. Thus, we may estimate the magnitude of the integrated inelastic (Hawking) differential cross section $d \sigma_{H} / d q^{2}$ by taking the result in Eq. (18) and multiplying it by a factor of $q v \sim r_{s}^{-1}$. We then find that, parametrically,

$$
\frac{d \sigma_{H}}{d \sigma_{N}} \sim \frac{q^{2}}{m_{\mathrm{Pl}}^{2}},
$$

up to factors $\left(r_{s} q\right)^{2}$, which we cannot determine by purely dimensional arguments and are treated as being of order unity for the purposes of this estimate. We see that inelastic scattering is a quantum gravity effect, of the same order in $q^{2} / m_{\mathrm{Pl}}^{2}$ as the one-loop correction to elastic scattering that arises from graviton vacuum polarization effects of the type first computed in [2] and illustrated in Fig. 2. Our result in

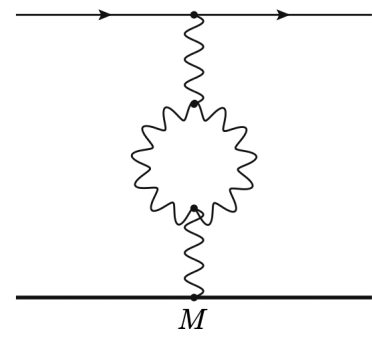

(a)

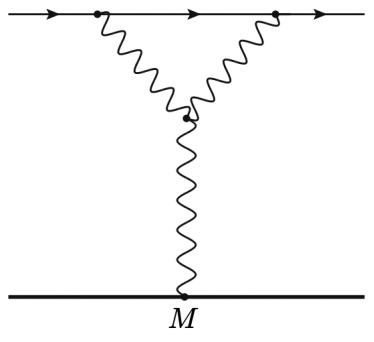

(b)
FIG. 2. Selected one-loop corrections to the elastic scattering process in perturbative quantum gravity. Diagram (a) contributes at first order in $q^{2} / m_{\mathrm{Pl}}^{2} \ll 1$, while (b) encodes both classical corrections of order $r_{s} E_{\phi} \ll 1$ and quantum effects of order $q^{2} / m_{\mathrm{Pl}}^{2}$. The top (arrowed) line corresponds to $\phi$, while the (bottom) solid line corresponds to the black hole, treated as static point source. 
Eq. (18) should then be interpreted as a new type of calculable, leading-order, quantum gravity effect in black hole quantum mechanics. Moreover, the prediction is made within a systematic expansion with calculable corrections.

Conclusions. - We have presented what, to our knowledge, is the first computation of quantum gravity effects in scattering processes that account for the black holes horizon fluctuations. Our approach relies on EFT methods presented in $[6,7]$. In this EFT, the leading quantum corrections due to horizon dynamics is represented by the exchange of virtual Hawking radiation. What is interesting about these effects is that, despite being nonperturbative in nature, they are not as suppressed as one might naively have expected. Instead, they scale in the same way in the $q^{2} / m_{\mathrm{Pl}}^{2}$ power counting as the more familiar one-loop graviton vacuum polarization [2] corrections to scattering that arise when treating the black hole sources as elementary particles (i.e., quantum fields).

A natural question to ask is how the inelastic scattering rate calculated here compares to on shell processes, e.g., radiative pressure. Given the on shell nature of the incoming graviton in this case and the fundamental (pointlike) nature of the scattered particle, such a process will necessarily be suppressed by further powers of $q^{2} / m_{\mathrm{Pl}}^{2}$ due to the existence of final state reradiation. Similarly, such a process would be suppressed in the case of a particle with nontrivial internal structure, even if no radiation appears in the final state.

In this Letter, we have only considered a simple inelastic process in which a scalar field scatters gravitationally off a 4D Schwarzschild black hole. However, our methods should apply more broadly to a larger class of scattering processes as well as to more generic black holes that may carry electric and magnetic charges and/or spin. Work on such generalizations is under way and will be presented in future publications.

This work was partially supported by the U.S. Department of Energy under Grants No. DE-SC0017660 (W. G.) and No. DE-FG02-04ER41338 and No. FG02-06ER41449 (I. Z. R.).

[1] J. F. Donoghue, Phys. Rev. Lett. 72, 2996 (1994); Phys. Rev. D 50, 3874 (1994).

[2] G. 't Hooft and M. J. G. Veltman, Ann. Inst. Henri Poincaré, A20, 69 (1974).

[3] M. H. Goroff and A. Sagnotti, Nucl. Phys. B266, 709 (1986).

[4] S. W. Hawking, Commun. Math. Phys. 43, 199 (1975); 46, 206(E) (1976).

[5] D. N. Page, Phys. Rev. D 13, 198 (1976).

[6] W. D. Goldberger and I. Z. Rothstein, J. High Energy Phys. 04 (2020) 056.

[7] W. D. Goldberger and I. Z. Rothstein, Phys. Rev. D 73, 104030 (2006).

[8] W. D. Goldberger and I. Z. Rothstein, arXiv:2007.00726.

[9] W. D. Goldberger and I. Z. Rothstein, Phys. Rev. D 73, 104029 (2006); Gen. Relativ. Gravit. 38, 1537 (2006).

[10] W. G. Unruh, Phys. Rev. D 14, 870 (1976).

[11] J. B. Hartle and S. W. Hawking, Phys. Rev. D 13, 2188 (1976).

[12] W. D. Goldberger and I. Z. Rothstein, arXiv:2007.00726.

[13] J. D. Bekenstein and A. Meisels, Phys. Rev. D 15, 2775 (1977).

[14] P. Panangaden and R. M. Wald, Phys. Rev. D 16, 929 (1977).

[15] R. Mertig, M. Bohm, and A. Denner, Comput. Phys. Commun. 64, 345 (1991); V. Shtabovenko, R. Mertig, and F. Orellana, Comput. Phys. Commun. 207, 432 (2016). 